\title{
3 Verlagssuche und Rezeption in Brasilien
}

\subsection{Kommentierter Überblick: Erstausgaben in Brasilien}

Die folgende Übersicht über Erstausgaben des literarischen Werkes Clarice Lispectors in Brasilien (ohne Kinderbücher, ohne Crônicas ${ }^{25}$, Interviews, Briefe) ermöglicht einen ersten Überblick ${ }^{26}$, auch über die Verlagswechsel in ihrer Publikationsgeschichte. Die kurzen Kommentare, die die Übersicht begleiten, bieten eine erste Orientierung zum Thema Verlagssuche und Verlagswechsel. Im Folgenden werden dann einige zentrale Episoden aus dieser Übersicht herausgegriffen und im Detail kommentiert.

Der erste Roman der damals 23-jährigen Autorin erschien bei A Noite und wurde in Brasilien als Sensation gefeiert:

1943 Perto do coração selvagem. Rio de Janeiro: A Noite.

Clarice Lispector hoffte, auch ihren zweiten Roman im gleichen Verlag unterbringen zu können, was ihr aber nicht gelang. Sie musste sich nach ihrer Hochzeit mit dem Diplomaten Maury Gurgel Valente 1943 und der Ausreise der beiden im darauffolgenden Jahr (zu den Stationen ihrer Auslandsaufenthalte vgl. Meyer-Krentler 2019: 62-68) nun vom Ausland aus um ihre Publikationen in Brasilien bemühen. Schließlich veröffentlichte der Verlag Agir den Text:

1946 O lustre. Rio de Janeiro: Livraria Agir Editora.

Der dritte Roman erschien erneut bei A Noite.

1948 A cidade sitiada. Rio de Janeiro: A Noite.

Einige Zeit später erschien Lispectors erster Band mit Kurzgeschichten in einer 52-seitigen Ausgabe des Ministério de Educação e Saúde:

25 Eine Sammlung ihrer Crônicas erschien erstmals unter dem Titel A descoberta do mundo 1984 bei Nova Fronteira - Lispector hatte diese sehr persönlich gehaltenen literarischen Kolumnen für das Jornal do Brasil verfasst und dort zwischen August 1967 und Dezember 1976 publiziert. Es bestehen teilweise sehr direkte thematische Zusammenhänge zu ihren literarischen Texten im engeren Sinne, manches spräche dafür, sie hier zu berücksichtigen. Aus Gründen der Übersichtlichkeit - insbesondere auch, was den noch folgenden Überblick über mehrere andere Sprachräume betrifft - sind sie in dieser Studie ausgeklammert.

26 Neben den Erstausgaben erschienen in mehreren Fällen innerhalb relativ kurzer Zeitspannen weitere Ausgaben desselben Titels in unterschiedlichen brasilianischen Verlagshäusern, die hier der Übersichtlichkeit halber nicht aufgeführt sind (vgl. dazu Marting 1993). 
1952 Alguns contos. Rio de Janeiro: Ministério de Educação e Saúde.

Danach vergingen viele Jahre, in denen Lispector große Schwierigkeiten hatte, Verlage für ihre Texte zu finden. Erst 8 Jahre später erschien die erste umfangreichere Sammlung mit Kurzgeschichten:

1960 Laços de família. Rio de Janeiro: Francisco Alves.

Dies ist die erste Erzählsammlung, die später ins Englische übersetzt wurde (für University of Texas Press, 1972 übersetzt von Giovanni Pontiero). Lispectors Ruhm als Autorin von Kurzgeschichten wird sich in erster Linie auf diesen Band gründen, aus dem zahlreiche Texte auch einzeln in Brasilien erschienen waren (in Zeitungen/Zeitschriften, u. a. Senhor). Im auf den Erzählband folgenden Jahr erschien auch endlich ihr Roman A maçã no escuro, um dessen Publikation sie lange gekämpft hatte.

1961 A maçã no escuro. Rio de Janeiro: Francisco Alves.

Auf diesen Band wurde der US-amerikanische Verleger Alfred A. Knopf aufmerksam und kaufte die Rechte. Es folgte eine im Hinblick auf die Verlagssuche und die Qualität der Ausgaben vergleichsweise entspannte Zeit, in der Lispectors Texte im neu gegründeten Verlag ihrer Freunde Fernando Sabino und Rubem Braga erschienen und sorgfältig verlegt wurden: Der Verlag hieß zunächst Editôra do Autor, dann nach einer Trennung der beiden Verleger von dem dritten Verlagsgründer ab 1967 Sabià. In dieser Phase erschien der 1963 entstandene Text A paixão segundo G. H., nach Meinung verschiedener brasilianischer Experten ihr bedeutendster Roman, der jedoch zunächst von der Literaturkritik fast gänzlich unbeachtet blieb.

1964 A paixão segundo G. H. Rio de Janeiro: Editôra do Autor.

Der darauffolgende Band vereint „contos e crônicas“:

1964 A Legião Estrangeira. Rio de Janeiro: Editôra do Autor.

Die darauffolgenden Texte erschienen dann bei Sabià:

1969 Uma aprendizagem ou O livro dos prazeres. Rio de Janeiro: Sabià. 1971 Felicidade clandestina. Rio de Janeiro: Sabià.

Sabià wurde 1972 an den Verlag José Olympio verkauft, Lispector gewann für ihren Text Áqua viva, einen der zentralen, längeren Erzähltexte ihres Schaffens, den Verleger Álvaro Pacheco von Artenova, der im folgenden mehrere Bände publizierte:

1973 Água viva. Rio de Janeiro: Editôra Artenova.

1973 A imitação da rosa. Rio de Janeiro: Editôra Artenova.

1974 A via crucis do corpo. Rio de Janeiro: Artenova.

1974 Onde estivestes de noite? Rio de Janeiro: Artenova. 
Auch diese Zusammenarbeit war nicht von Bestand und endete mit einem Zerwürfnis zwischen Autorin und Verleger (vgl. Kap. 3.2). Der letzte zu Lebzeiten publizierte Roman Lispectors - der zu einem Erfolg insbesondere bei der US-amerikanischen Literaturkritik werden sollte - erschien bei José Olympio.

1977 A hora da estrela, Rio de Janeiro: José Olympio.

Postum veröffentlichte ihre Lektorin und Vertraute Olga Borelli Lispectors letzten Prosatext bei Nova Fronteira:

1978 Um sopro da vida: Pulsações. Rio de Janeiro: Nova Fronteira.

Dieser Text entstand zwischen 1974 und 1977 und ist der einzige postum veröffentlichte, längere Erzähltext Lispectors. Vorangestellt ist ein Kommentar Olga Borellis. Ihr zufolge entstand der Text gleichzeitig mit dem letzten zu Lebzeiten entstandenen Roman A hora da estrela. Lispector habe, so Borelli, an Um sopro da vida bis zu ihrem Tod gearbeitet.

Bezüglich Um sopro da vida, so lässt sich nachlesen, gelte es demnach in besonderer Weise als unklar, wie „vollständig“ oder „abgeschlossen“ der Text sei (vgl. Marling 1993: 143). Die verlegerische Meinung, Lispectors Texte seien nicht vollständig bearbeitet und man könne unautorisierte Veränderungen daran vornehmen, hat allerdings auch die Übersetzungs- und Publikationsgeschichte anderer Texte ihres umfangreichen, nach innovativen ästhetischen Maßstäben entwickelten Werkes geprägt, wie die folgenden Kapitel zeigen.

\subsection{Clarice Lispectors aufreibende Verlagssuche in Brasilien}

Um Eindrücke davon zu vermitteln, was die Verlagssuche in Brasilien für Clarice Lispector bedeutete, werden hier drei entscheidende Phasen herausgegriffen und detailliert besprochen. Im Kontext der Themen des vorliegenden Bandes werden 1. ihre Publikationsschwierigkeiten in den 1950er Jahren geschildert, 2. ihr erster Publikumserfolg bei Senhor ab 1959 herausgegriffen, der mit der Rückkehr der Autorin nach Brasilien einherging, und 3. die verlegerische Situation 1975/76 wenige Jahre vor Clarices Tod. Alle drei Stationen zeugen davon, wie aufreibend die Verlagssuche für Clarice Lispector in ihrem eigenen Sprachraum, in ihrer Heimat Brasilien für sie war - was leicht in Vergessenheit gerät, wenn man bedenkt, wie sehr sie in Brasilien heute verehrt wird. Die Recherche zum Thema Verlagssuche speist sich aus unterschiedlichen Quellen, in besonderer Weise jedoch aus Benjamin Mosers fast ein Jahrzehnt umfassenden Recherchen im Zuge der Vorbereitung seiner 2009 erschienenen Biographie, die im Hinblick auf die hier dargestellten Inhalte besonders wertvoll sind. 


\section{Publikationsschwierigkeiten im Brasilien der 1950er Jahre}

Im März 1956 beendete Clarice Lispector die Arbeit an A maçã no escuro nach einem langen und mühsamen Entstehungsprozess - Moser schreibt von 11 Entwürfen (Moser 2013a: 287) des in der Erstausgabe bei Rocco (1961) 336-seitigen Bandes. Sie schickte Kopien an Érico Veríssimo und Fernando Sabino, der sich darum bemühte, das Manuskript an einen passenden Verlag zu vermitteln und im Juni desselben Jahres die Nachricht übermittelte, dass Ênio Silveira von Civilização Brasileira das Buch im Oktober oder November herausbringen wolle (vgl. Brief vom 7. Mai 1956 von Clarice Lispector an Fernando Sabino und Antwortbrief vom 8. Juni 1956, in: Lispector/Sabino 2016: 139-142). Der Verleger, der gerade große Erfolge mit Fernando Sabinos Schwarzer Mittag feierte, war zunächst begeistert (Lispector/Sabino 2016: 141), allerdings verstrich der geplante Publikationstermin aus offenbar auch für Fernando Sabino undurchsichtigen Gründen (vgl. Brief von Fernando Sabino an Clarice Lispector vom 26.9.1956, in: Lispector/Sabino 2016: 161). Moser verweist darauf, dass das Verlagsgeschäft in dieser Zeit in Brasilien eigentlich boomte: „Bis 1945 hatte man in Brasilien durchschnittlich zwanzig Millionen Bücher pro Jahr veröffentlicht; diese Zahl sollte sich bis $1962 \mathrm{mehr}$ als verdreifachen: auf sechsundsechzig Millionen“ (Moser 2013a: 288). Silveira kommentierte ungeachtet dieser Entwicklungen dann wohl später, das Buch werde nicht leicht zu verkaufen sein, aus Prestigegründen werde er es aber veröffentlichen und wolle es spätestens bis Juni 1957 herausbringen (Moser 2013a: 288, FN 7). Clarice Lispector gefiel dieser Umgang mit ihrem Text gar nicht, die unsichere Warteposition, die für sie damit einherging, widerstrebte ihr. Dies ist natürlich ohnehin verständlich, für Clarice Lispector bedeuteten solche Erlebnisse aber offenbar eine besondere Belastung, eine Einschränkung in ihrer Art, zu arbeiten.

Aus den Unterlagen in ihrem Archiv in der Fundação Casa de Rui Barbosa in Rio de Janeiro geht, Marlene Gomes Mendes' Arbeit zufolge, hervor, dass sie sich nicht mehr mit Texten beschäftigte, die sie aus der Hand gegeben hatte (Gomes Mendes 2007). Mit einer einzigen Ausnahme (Agua viva) bewahrte sie keine Original-Manuskripte $\mathrm{zu}$ ihrem fiktionalen Werk auf. $\mathrm{Zu}$ ihrer Arbeitsweise passte es daher am besten, Texte schnell in einem Verlag unterzubringen, nachdem sie fertiggestellt waren, und sie dann nicht mehr zur Hand zu nehmen. Abgesehen davon, dass für jede Autorin und jeden Autor das Warten auf eine verschobene Publikation belastend ist, muss es für Lispector eine besondere Einschränkung ihrer Arbeit an neuen Texten bedeutet haben, fertiggestellte nicht publizieren zu können. Lispectors Arbeitsprozess betreffend, übersetzt Gomes Mendes in ihrem Vorwort zu der spanischen Ausgabe Para no olvidar mit verschiedenen Crônicas und anderen Kurztexten der Autorin einen 
Kommentar Lispectors zu diesem Thema, leider ohne Nachweis des Originals. Er stammt offensichtlich aus einem Brief an Olga Borelli (mit der die Autorin sich in ihren letzten Lebensjahren sehr detailliert $\mathrm{zu}$ ihren Texten beriet): „... No he podido esperarte, me estaba muriendo de cansancio, porque he estado trabajando sin interrupción desde las cinco de la mañana. Desgraciadamente tengo que hacer yo la copia de Detrás del pensamiento, siempre he hecho la última copia de mis libros anteriores por que cada vez que copio voy modificando, aumentando, en definitiva modificando.“ (zitiert nach Gomes Mendes 2007: 13-14). ${ }^{27}$ Mendes zitiert weiterhin aus einem Gespräch Lispectors mit Affonso Romano de Sant'Anna vom 20. Oktober 1976 (ein Interview im Auftrag des Museu da Imagem e do Som, Gomes Mendes 2007: 14). Der Interviewer: „Tienes tus textos escritos en la cabeza. Y una vez dijiste una cosa impresionante: que nunca relees un texto tuyo.“ Daraufhin Clarice Lispetor: „No. Me da náuseas. Cuando se publica es un libro muerto. No quiero saber nada más de él.“ Olga Borelli, so Mendes, habe diese Haltung Lispectos auf Anfrage bestätigt: „Clarice no revisaba sus textos después de haber mandado los originales a la editorial“ (Gomes Mendes 2007: 14).

Während Clarice Lispector 1957 vergeblich auf die Publikation von A maçã no escuro wartete und sich über das Verhalten von Ênio Silveira ärgerte, gab sie eine Fassung des Manuskriptes Rubem Braga, der sie in Washington besuchte und es, zurück in Brasilien, dem renommierten Verlag José Olympio in Rio zur Publikation vorschlug - mit der Reaktion, man wolle es „sofort veröffentlichen“ (Moser 2013a: 288). Aber es gab keine konkrete Zusage. Lispector wollte nun nicht länger warten und erwog sogar, das Buch im Selbstverlag zu veröffentlichen - was laut Benjamin Moser aus ihrer Bitte an Fernando Sabino hervorgehe, den Kontakt zu Druckereien herzustellen (Moser 2013a: 288). Moser beschreibt, wie entmutigt Lispector zu dieser Zeit durch die so schwierige Verlagssuche in Brasilien gewesen sei, zumal es ähnliche Schwierigkeiten mit mehreren Projekten gab. 1954 hatte sie von Fernando Sabinos Freund José Simeão Leal, der bereits Alguns contos publiziert hatte, erstmals einen Vorschuss für einen literarischen Text erhalten: Er hatte einen vollständigen Erzählband in Auftrag gegeben, an dem Lispector parallel zu A maçã no escuro schrieb, er wurde im März 1955 fertig: Laços de família, ein Text, der später zu den wichtigsten Texten der Autorin gezählt werden sollte, der zunächst aber nur von einzelnen Freunden begeistert aufgenommen wurde, während sich die Publikation auch hier auf undurchsichtige Weise verzögerte und der Text nicht an ein breiteres Publikum gelangte. Im Juni 1956 informierte der Verleger Fernando Sabino, der in Brasilien weiterhin als Clarices Agent auftrat,

27 Detrás del pensamiento ist die spanische Übersetzung des Arbeitstitels für den später als Aqua viva (Agua viva) publizierten Text. 
darüber, dass das Buch bereits gesetzt sei. Ein knappes Jahr später war es immer noch nicht erschienen. Im März 1957 bemühte sich erneut Rubem Braga, in der Sache zu helfen und die Rechte zurückzuholen, um die Geschichten in der Zeitung $O$ Estado de S. Paulo unterzubringen (Moser 2013a: 289). Gleichzeitig bot Fernando Sabino sie dem Verlag Agir an, der bereits Clarices Lüster publiziert hatte. Dann, so schildert Moser weiter die Ergebnisse seiner diesbezüglichen Recherchen, vergingen „weitere demütigende anderthalb Jahre“. Anderthalb Jahre! Und dies während, so Moser, Civilização Brasileira sonst sehr produktiv war und nur wenige Jahre später nachweislich an jedem Arbeitstag einen neuen Titel publizierte. Es wiederholte sich dann die Geschichte: Érico Veríssimo fand einen neuen brasilianischen Verlag für beide Bücher: Globo. Leal wollte die Originale der Erzählungen, die bereits im Druck seien, aber auf keinen Fall herausgeben, und gerade, als man bei Globo für den Roman zugesagt hatte, tauchte dieser im Katalog von Civilização Brasileira auf. Der angekündigte Termin verstrich aber wieder, ohne dass das Buch publiziert wurde. „Das erschöpfende Hin und Her der Erwartungen und der enttäuschten Hoffnungen und die niederdrückende Tatsache, im mittleren Alter und in der Mitte ihrer Karriere gezwungen zu sein, um einen Verlag zu betteln, taten Clarices Gemütsverfassung nicht gut“, kommentiert Moser (Moser 2013a: 289).

So also sah die Situation in Brasilien für Clarice Lispector in den für sie so wichtigen 1950er Jahren aus, in denen erstmals auch ein Text von ihr ins Ausland übersetzt werden sollte. Später entspannte sich die Situation, zunächst durch die Verlagsgründung der Editora do Autor ihrer Freunde Fernando Sabino und Rubem Braga im Jahr 1960, die nach einem Streit mehrerer Verlagsleiter ab 1966 gemeinsam den Verlag Sabià führten, dann auch durch ihren zunehmenden Bekanntheitsgrad in Brasilien. Verschont blieb sie aber zeitlebens nicht von dieser Art von Auseinandersetzungen um Publikationsmöglichkeiten und den damit verbundenen Zweifeln an ihren Texten, denen ein Element des Offenen, des oftmals als unvollendet Missverstandenen inhärent war. $\mathrm{Zu}$ Affonso Romano de Sant'Anna hatte sie nur ein Jahr vor ihrem Tod zu Re-Lektüre ihrer Texte gesagt: „Me da náuseas. Cuando se publica es un libro muerto. No quiero saber nada más de él. Y cuando lo leo me suena extraño, me parece malo. No los leo, iqué va!“ (zitiert nach Gomes Mendes 2007: 14). 


\section{Erster Publikumserfolg bei Senhor ab 1959 und Clarices Rückkehr nach Brasilien}

In den geschilderten Jahren zwischen 1956 und 1958 muss Clarice Lispector sehr unter ihrer Isolation, unter der immer wieder scheiternden Verlagssuche gelitten haben. Am 12. Juli 1956 schrieb sie an Fernando Sabino: „Ich erlebe gerade etwas sehr Unerfreuliches. Je mehr Zeit vergeht, desto mehr bekomme ich das Gefühl, dass ich an keinem Ort lebe, und dass kein Ort ,mich will'.“ (Brief an Fernando Sabino vom 12.7.1956, in: Lispector/Sabino 2016: 145). Moser kommentiert dies in seiner Biographie so:

Die traurige Wahrhheit war, dass Clarice teilweise recht hatte. Außerhalb des Kerns von
Künstlern und Intellektuellen, die seit dem Erscheinen von Perto do coração selvagem
mehr als ein Jahrzehnt zuvor von ihr fasziniert waren, hatte man Clarice inzwischen fast
völlig vergessen. Ihre späteren Romane hatten den Ruhm, den sie sich mit dem ersten ver-
dient hatte, nicht vermehrt; zudem lebte sie seit vielen Jahren im Ausland. Sie hatte kei-
nen Namen mehr, und wie die Briefe, die sie von Fernando erhielt, immer deutlicher
machten, brannte kein Verlag darauf, sich auf eine schwierige, vierhundert Seiten lange
kabbalistische Allegorie von einer unbekannten Autorin einzulassen, gleichgültig, wel-
chen Ruf sie bei gewissen Intellektuellen genoss.
(Moser 2013a: 290)

Diese Situation veränderte sich 1958 ein wenig, als sie eine Anfrage von dem jungen Journalisten Nahum Sirotzky erhielt, einem Cousin ihres Freundes Samuel Wainer, der eine neue Kulturzeitschrift herausbringen wollte, die in den darauffolgenden Jahren in Brasilien sehr anerkannt werden würde: Senhor. In Abgrenzung zu bestehenden Zeitschriften wie Dom Casmurro und mit Blick auf die großen ausländischen Organe wie den New Yorker würden Texte dort redigiert werden, so schwebte es Sirotzky vor. Paulo Francis, der für diese redaktionelle Arbeit in der Sparte Literatur zuständig war, kommentierte einmal, wie weitreichend dieser Wandel gewesen sei. Die fruchtbare Zusammenarbeit mit Clarice Lispector, die nicht nur in der ersten Ausgabe vertreten war, sondern fortan sehr regelmäßig und die seit 1961 eine Kolumne für jedes Heft schrieb, ist nicht zuletzt wohl auf ihr Verhältnis zu ihren Texten zurückzuführen, auf eine Offenheit für das Überarbeiten und Ergänzen eigener Texte mit den verschiedenen Überarbeitungsgängen. Paulo Francis schreibt darüber: „Clarice reagierte völlig normal und schrieb manchmal Passagen um, die, wie sie einräumte, unklar waren. In der brasilianischen Literatur ist das tabu ... man rührt die Texte der großen Namen nicht an“ (Francis 1987). Bei Senhor passierte nun interessanterweise etwas in gewisser Weise Umgekehrtes im Vergleich zu den Verhältnissen in der brasilianischen Verlagsbranche: Während die Zusammenarbeit mit Lispector funktionierte und sie sehr regelmäßig publiziert wurde, lehnte die Zeitschrift mit ihren strengen Qualitätsansprüchen an hervorragende künstlerische Leistungen namhafte oder 
einflussreiche Autoren mitunter ab, wie Érico Veríssimo in einem Fall oder den späteren brasilianischen Präsidenten Jânio Quadros, der literarische Ambitionen hatte und mehrere Artikel einreichte, wie Moser berichtet (Moser 2013a: 290). Seiner Darstellung zufolge war die Publikation in Senhor „die erste Begegnung mit echter Popularität“ für Lispector, sie wurde zur Inspiration für Leser/innen und insbesondere auch für Künstler/innen verschiedener Sparten, die einen „modernen“ Stil, eine neue Ästhetik suchten und in mehreren Fällen Lispector genauso als Einfluss nannten wie João Gilberto und den Bossa Nova. Ungefähr zu der Zeit, so schreibt Moser, als ihre ersten Erzählungen bei Senhor erschienen, traf sie „Vorbereitungen, ihren Mann zu verlassen und, diesmal endgültig, nach Rio de Janeiro zurückzukehren“ (Moser 2013a: 290). Damit ging einher, dass sie sich fortan nicht mehr aus der Ferne und mittels Vermittlerfiguren um ihre Publikationen kümmern musste. Wie stark die Situation, in der sie als Autorin war - einerseits in Vergessenheit geraten zu sein und größte Schwierigkeiten zu haben, ihre Manuskripte in Verlagen unterzubringen, andererseits aber durch die Erfahrungen mit Senhor zu erleben, dass eine Veränderung, ein „gesehen werden“ möglich wäre, zu diesem Entschluss beigetragen haben mögen, lässt sich nicht sagen. Nur dass sich Lispector in genau dieser Konstellation zu einer Rückkehr entschloss, ist eine Tatsache: In einer Konstellation, in der sie sich von Brasilien schmerzlich getrennt und als Autorin nicht beachtet fühlte, erstmals aber auch in einem wichtigen Punkt gesehen: Sirotzky hatte ihr in seinem ersten Brief geschrieben: „Wir möchten gern Ihre Erzählungen lesen, die wir nie für verständlich gehalten haben“ (Brief von Nahum Sirotzky an Clarice Lispector, 12.11.1958, zitiert nach Moser 2013a: 290).

\section{Die verlegerische Situation 1975/76}

Was geschah nun, nachdem Lispector sich 1959 von ihrem Mann scheiden ließ und nach Rio de Janeiro zurückkehrte, hinsichtlich der Publikationsprobleme mit ihren Texten? Die gesamte Publikationsgeschichte Lispectors in Brasilien kann und soll hier nicht für jeden Band aufgearbeitet werden. Um die lebenslangen Schwierigkeiten Lispectors mit brasilianischen Verlegern in den Blick zu nehmen, ist es aber wesentlich, zu sehen, was in den Jahren ihres größten Erfolges zu Lebzeiten in Brasilien, in der Zeit ab der Jahreswende 1975/76 geschah. Moser bezeichnet das Ende des Jahres 1975 als „Ende eines aufreibenden Jahres, in dem sie wie so oft Schwierigkeiten mit Verlegern gehabt hatte“ (Moser 2013a: 441). Er berichtet von einer Publikation im Verlag Artenova mit dem Verleger Álvaro Pacheco, der eine Sammlung der Interviews herausgebracht hatte, die sie im Laufe der Jahre mit berühmten brasilianischen Persönlichkeiten geführt hatte. Pacheco 
war laut Mosers Darstellung in Clarices Augen Jahre zuvor ein Wagnis eingegangen, als er Aqua viva publizierte, weshalb sie sich ihm verbunden fühlte, dann allerdings zunehmend unzufrieden wurde wegen der mangelnden Sorgfalt, mit der ihre Bücher bei Artenova publiziert wurden. „Aber Clarice drückte ein Auge $\mathrm{zu}$, bis sie das Gefühl hatte, um ihre Tantiemen betrogen zu werden“, schreibt Moser. Nach den Aussagen von Alberto Dines habe es dem Verleger, der mehr eine Druckerei als einen Verlag betrieben habe, widerstrebt, Autoren für ihre Arbeit zu bezahlen: „Er glaubte, Schriftstellern dadurch, dass er sie veröffentlichte, einen Gefallen zu tun“ (Moser 2013a: 441). Die Autorin Lispector sei, was auch die Biographie von Nadia Battella Gotlib (1995) nahelegt, geschäftlich trotz ihrer juristischen Ausbildung recht inkompetent gewesen: „Aber nun, als ihre Honorarabrechnungen beleidigend niedrig wurden, roch sogar sie Lunte“ (Moser 2013a: 442). Dass sie nie von der Literatur habe leben können, führte Lispector selbst auf einen kontinuierlichen Missbrauch der Verleger an ihrem Werk zurück (Battella Gotlib 2007: 436-437) - auch noch, als sie zu den bekanntesten Schriftsteller/innen Brasiliens zählte und Übersetzungen in Portugal, Frankreich und den USA vorlagen.

Olga Borelli (1981) berichtet, wie auch Moser (2013a) und Manzo (1998), von der Szene, die sich im Winter 1975/76 im Verlag abspielte, als sie Pacheco um ein Gespräch bat - im gleichen Jahr, in dem Lispector in Brasilien große Erfolge feierte und ungeheuer populär wurde. Pacheco hatte sie im Verlag zuerst lange warten lassen, um ihr dann 140 Cruzeiros in bar auszuhändigen, ihre Tantiemen für ein halbes Jahr und alle fünf bei Artenova erschienenen Bücher. Olga Borelli schreibt, sie habe Clarice Lispector noch nie so wütend gesehen: sie sei hinausgelaufen und habe das Geld einem Bettler geschenkt (Borelli 1981: 47-48).

Erst als ihre Freundin und Schriftstellerkollegin Nélida Piñon sie mit der Literaturagentin Carmen Balcells in Barcelona zusammenbrachte, die bekanntlich bereits zu dieser Zeit viele lateinamerikanische Autor/innen aus dem spanischsprachigen Bereich sehr erfolgreich vertrat, wurde Clarice Lispector erstmals professionell vertreten - ihre positiven Publikationserfahrungen in Brasilien in der Zeit davor, etwa bei der Editora do Autor und später bei Sabiá, waren bis dahin immer nur vereinzelte. Durch ihre Krebserkrankung und ihren frühen Tod 1977 konnte Clarice Lispector selbst die Vorteile eines professionellen Umgangs mit ihrem Werk in der Verlagsbranche allerdings nicht mehr genießen.

\subsection{Zur Rezeption in Brasilien}

In literaturgeschichtlichen Darstellungen des späten 20. und beginnenden 21. Jahrhunderts zu Brasilien erfolgt oftmals eine scheinbar problemlose Einordnung 
Lispectors in die brasilianische Literaturgeschichte. Antonio Maura liefert in seiner gerade auch was Charakteristiken von Lispectors Schaffen betrifft sehr wertvollen Cartografia Literaria de Brasil (2014) eine solche: Seiner Darstellung zufolge seien brasilianische Autor/innen Mitte des 20. Jahrunderts in zwei Hauptströmungen einteilbar. Zum einen ist da die „novela regionalista“, zum anderen „una narrativa de carácter psicológico-existencial“, der er Lispector zusammen mit Autoren wie Lucio Cardoso, Lygia Fagundes Telles und Osman Lins zurechnet. Luisa Trias Folch geht in ihrer Literatura brasileña noch einen Schritt weiter und parallelisiert gewissermaßen Lispector mit der nur 3 Jahre jüngeren Lygia Fagundes Telles: die beiden teilen, so Trias Folch, „el análisis psicológico, realizado a través de técnicas como el flujo de conciencia y el monólogo interior" (Trias Folch 2006: 309) - die sehr grundlegenden Unterschiede zwischen dieses Autorinnen, die den Vergleich problematisch machen, bleiben dabei unterbelichtet. Neben den genannten Grundströmungen nennt Maura, als dritten Punkt, „a medio camino“: João Guimarães Rosa „que explora tanto el aspecto regional-mítico del sertão mineiro como ahonda en la profunda significación de la vida humana“ (Maura 2014: 85). Nach anderen Darstellungen, z. B. von José Paulo Paes gehört Lispector in erster Linie zur so genannten 45er-Generation, das heißt, zu einer Gruppe brasilianischer Autorinnen und Autoren, die nach dem 2. Weltkrieg zu publizieren beginnen (unter anderem: Clarice Lispector, Murilo Rubião, José J. Viega, Osman Lins) und die sowohl von den Erfahrungen der Kriegsjahre als auch von den französischen Existenzialisten beeinflusst seien (vgl. Maura 2014: 85-86). All dies mag hilfreich sein, um die Autorin grob in ihrem Kontext zu verorten, aber es darf nicht darüber hinwegtäuschen, dass Lispector lange Jahre in Brasilien als völliger Sonderfall wahrgenommen wurde.

Márgara Russotto hat in ihrer Essaysammlung Sustentación del enigma. Cuatro ensayos sobre Clarice Lispector (2013) Rezeptionslinien herausgearbeitet, die gerade mit Blick auf die frühe Rezeption in Brasilien und die dadurch geprägten Vorbedingungen für eine internationale Rezeption sehr interessant sind. Russotto beschreibt die Sonderposition von Lispectors Werk im brasilianischen Kontext ausgehend von einigen Grundzügen ihres literarischen Denkens: „Para Clarice, como se sabe, la representación misma constituye un territorio en crisis y un acto fallido en todos los casos. Por lo tanto, el poder de la mimesis es constantemente problematizado" (Russotto 2013: 21-22). Russotto verweist auf eine Erzählung Lispectors, die nur aus einem einzigen Satz besteht: „Tinha uma vez um pássaro, meu Deus!“, um dann auszuführen:

Si la descripción de un pájaro es imposible, siquiera como un simple ejercicio escolar y convencional, ¿cómo representar la complejidad del mundo? La confianza en la representación y en sus respectivos instrumentos es algo completamente ajeno al pensamiento 
Clariceano. El artificio la enerva, y el lenguaje se le presenta siempre con el rostro desfigurado y atormentado de sus propios límites y perplejidades.

(Russotto 2013: 22)

Vor diesem Hintergrund sei es verständlich, so Russotto, dass im Kontext der Erwartungen der brasilianischen Literaturkritik in den 1940er Jahren, die von politischen und sozialen Sorgen und Fragen geprägt waren, und auch vor dem Hintergrund des kritischen Realismus der 1930er Jahre, dass man da das Werk Lispectors als ,anders' deklarierte. Ästhetisch wie ideologisch sei ihr Schaffen zudem weit von den Stereotypen entfernt gewesen, die die internationale Kritik Literatur aus Brasilien zugeschrieben habe. Rusotto führt aus:

Debido a la vigencia de dichos estereotipos, parcialmente internalizados por buena parte de la crítica local como suele ocurrir en la dinámica de las relaciones interliterarias, su primera novela, Perto do Coração Selvagem de 1943, fue percibida bajo una óptica extraña, como si se hubiese gestado en terreno foráneo, ajeno a los grandes núcleos del imaginario brasileño con su carácter diferenciado dentro del conjunto de las literaturas del mundo. (Russotto 2013: 22)

Nach Russotto hätte es einen zu großen Widerspruch zu diesen tradierten Stereotypen bedeutet, hätte man Lispector stärker im Kontext der zeitgenössischen Literaturen aus Brasilien verortet. Sie beschreibt die Anknüpfungsmöglichkeiten von Lispectors Literatur zu anderen brasilianischen Texten und die Ähnlichkeiten oder Verbindungslinien, die durchaus bestanden. So sei es im brasilianischen Kontext der Zeit Kritiker/innen offenbar nicht möglich gewesen, den Debutroman innerhalb der brasilianischen Literatur zuzuordnen:

No a los movimientos mesiánicos protagonizados por colectividades surgidas de mundos aislados; ni a la gesta anónima de cangaceiros, retirantes, iluminados, y tantos otros personajes colectivos perdidos en los enormes espacios de la pre-modernidad brasileña. Y aunque el tiempo demostraría lo contrario con la llegada de la nordestina Macabea en $A$ hora da estrela (1977), nada parecía vincularla a las formas monumentales de dicha tradición, a la guerra por la tierra de Euclides da Cunha, a las sensualidades inestables y atormentadas de José Lins do Rego, a los humillados y ofendidos de Graciliano Ramos. Tampoco podía vincularse a esa picaresca urbana que ofrecían, cada uno a su modo, los relatos del desarraigo urbano y existencial de João Antonio, Rubem Braga, Carlos Drummond de Andrade, Fernando Sabino y otros, en una suerte de crónicas de la "supervivencia malandra”. Para esto último le faltaba el necesario sentido de superioridad irónica ante la desorientación moderna, y le sobraba patetismo y grotesco.

(Russotto 2013: 23)

Russotto kommt zu dem Schluss, die Erwartungen und Vorprägungen der brasilianischen Literaturkritik seien so bestimmend gewesen, dass das Werk von Clarice Lispector "un verdadero problema de la clasificación“ darstellte: „Ni historia, ni geografía, ni psicología, ¿cual era entonces el rostro de Clarice?” (Russotto 2013: 23) So kam es, dass viele Kritikerinnen und Kritiker, wie Sergio Milliet, ihr Abseitigsein, ihre Isolation und Andersartigkeit herausstellten: 
Essa harmonia preciosa e precisa entre a expressão e o fundo, a autora a alcançou magistralmente. De uma maneira que só observamos até agora em certos escritores franceses o ingleses, num Gide, num Morgan, por exemplo, o que de resto não implica em comparações deslocadas, muito embora, o romance de estréia de Clarice Lispector a eleve de chofre a um plano de absoluto destaque em nossa literatura.

(Milliet 1987: 4)

Eine Zeitlang, so Russotto, sei diese Randseitigkeit als formale Unzulänglichkeit missverstanden worden (Russotto 2013: 24). Jahrzehntelang sei von Seiten nicht nur der brasilianischen Kritik das Augenmerk auf das Unvollkommene, auf die Ambiguitäten gelegt worden, immer wieder ging es um vermeintlich nicht realisierte oder unvollkommene Aspekte ihrer Arbeiten.

In diesem Kontext des Unklassifizierbaren und dramatisch Vereinzelten habe man sie in der literarischen Welt von James Joyce - „por la presencia reiterada de lo epifánico“, von Virginia Woolf - „por el uso indeterminado y relacionante del tiempo“ und von Franz Kafka verortet -,,por la doble marca del absurdo y la parábola al mismo tiempo“ (Russotto 2013: 24). Außerhalb Brasiliens war diese Perspektive besonders stark, was nicht weiter erstaunlich ist, war dort doch das Wissen um brasilianische Literaturen viel weniger verbreitet als unter brasilianischen Kritiker/innen und Kommentator/innen. Aber auch Brasilien-Experten wie Earl Fitz (Vanderbilt) schreibt Russotto dieser Perspektive auf Lispectors Schaffen zu, unter anderem bezüglich der Einflüsse von Gide, Hesse und Djuna Barnes. Benjamin Moser stellt in seiner Biographie heraus, dass und warum bereits Lispectors Debutroman ungewöhnlich selten mit den Werken anderer brasilianischer Autor/innen verglichen wurde:

Es ist bemerkenswert, wie selten Kritiker das Buch mit denen anderer brasilianischer Schriftsteller verglichen. Stattdessen erwähnten sie Joyce, Virginia Woolf, Katherine Mansfield, Dostojewski, Proust, Gide und Charles Morgan. Das lag nicht einfach daran, dass die ganze Frage Brasiliens - jener ,sichere Instinkt für Nationalität‘, den Machado de Assis für den Kern der brasilianischen Literatur hielt - in Nahe dem wilden Herzen fehlt. Es lag daran, dass die Sprache des Romans nicht brasilianisch klang. [...] Später sollte sich diese Sprache als die einer großen brasilianischen Künstlerin einbürgern, doch zunächst klang sie exotisch.

(Moser 2013a: 165)

Diese Grundtendenz der Verortung ihres Werkes außerhalb der brasilianischen Literatur lässt sich sicher bestätigen, auch wenn es Ausnahmen gegeben hat, wie Antonio Candido, der eine (ausschließliche) Bezugnahme auf Einflüsse jenseits der brasilianischen Tradition bereits vermied, als er den Debutroman besprach (Candido 1945). Im Kontext einer solchen Linie der Interpretation innerhalb des brasilianischen Kontextes ist auch eine sehr treffende Einschätzung zu sehen, die Candido 1988 in einem Text über Clarice Lispectors Werk vornahm: 
Ora, em 1943 e 1946 apareceram dois escritores que retomaram o esforço de invenção da linguagem, coisa rara e perigosa, que quando dá certo eleva o perfil das literaturas: Clarice Lispector e João Guimarães Rosa. Para eles, o problema parecia consistir em obter um equilíbrio novo entre tema e palavra, de modo que a importância de ambos fosse igual.

(Candido 1988: XVIII)

Eine weitere, sehr bemerkenswerte Beobachtung schildert Russotto, wenn sie schreibt, es habe bei einer Reihe von Kritikerinnen und Kritikern die Tendenz gegeben, immer wieder auf Lispectors Schaffen zurückzukommen, indem man eigene Forschungsansätze revidiere oder in ungewöhnlich intensiver Weise neu bearbeite und vertiefe:

[...] en los casos de Benedito Nunes, Olga de Sá, Berta Waldman, Vilma Arêas y Affonso Romano de Sant'Anna, entre muchos otros, parece darse un cierto estado de constante insatisfacción interpretativa que obliga a relecturas correctivas o más profundas, como si hubiera siempre un excedente por desplazar a nuevas lecturas de efectos retroactivos y resemantizadores.

(Russotto 2013: 25)

Die „consagración canónica definitiva“ (Russotto 2013: 26) in Brasilien sei mit der Publikation der - tatsächlich hervorragenden und sehr umfassenden Biographie von Nadia Battella Gotlib eingetreten: Clarice - Uma Vida que se Conta. 1995 erschien diese Biographie in drei Auflagen. Der Effekt, dass das so bedeutsame wie schwer zu fassende literarische Werk Lispectors erst durch die Publikation einer Biographie wichtige und weiterreichende Aufmerksamkeit gewinnen konnte, war später auf internationaler Ebene noch einmal zu beobachten, als Benjamin Moser 2009 seine Biographie Why this World (Oxford University Press) publizierte und als Finalist des renommierten National Book Critics Circle Award in den USA ausgezeichnet wurde, was in den Feuilletons viel Beachtung fand - weit über den englischsprachigen Raum hinaus.

\subsection{Zur Rezeption im spanischsprachigen Lateinamerika}

Während Russotto für die Rezeption zu Lebzeiten der Autorin und insbesondere in Brasilien gewisse Linien ausmacht, die orientierend wirken, beschreibt sie die internationale Rezeption im Detail, ohne eine klare Entwicklung solcher Linien zu konstatieren. Es würde zu weit gehen, all die verschiedenen Ansätze der internationalen Kritik in den verschiedenen Sprachräumen nebeneinanderzustellen, wie sie sich bei Russotto findet (Russotto 2013: 27-38). Zwei Beobachtungen zur Rezeption in Lateinamerika, die auch darüber hinaus prägend waren, sollen hier aber herausgegriffen werden. Das spanischsprachige Lateinamerika, und insbesondere Argentinien, sind die Regionen, in denen Lispector 
neben Brasilien selbst ein teilweise vergleichbares Renommee als Autorin erlangte. Wichtig ist bei Russotto zum einen eine Bemerkung, die sich auf weibliche Autorschaft und Einflüsse unter Autorinnen bezieht: „En Hispanoamérica empieza el solitario diálogo entre iguales: la secreta y mutua inspiración entre escritoras que es un rasgo específico de filiación de género, un aspecto de la autoría femenina que va evidenciando un canon diferenciado en constante problematización” (Russotto 2013: 28). Das wirkungsmächtigste und bekannteste Beispiel für weibliche Netzwerkbildung entsteht, wie bereits angesprochen, in Frankreich (vgl. Kap. 4.1) - mit allen Vor- und Nachteilen einer Rezeption im Kontext des französischen Feminismus. Für die Netzwerkbildung in Lateinamerika sind vergleichbare negative Effekte nicht belegt worden - hier erschien Lispectors Werk allerdings auch nicht in reinen Frauenbuchverlagen.

Die zweite interessante Beobachtung von Russotto in diesem Zusammenhang bezieht sich auf die besondere Förderung Lispectors durch zwei Einzelpersonen, die als wichtige Gatekeeper im Kontext des Boom spanischsprachiger Literatur aus Lateinamerika ab den 1960er Jahren gelten. Sie schreibt: „Dos críticos uruguayos comentan y difundan su obra de manera distinta: Emir Rodríguez Monegal y Ângel Rama“ (Russotto 2013: 28, vgl. auch Strausfeld 1984). Die große Einflussnahme von Schlüsselfiguren auf die Zirkulation und (internationale) Rezeption schriftstellerischer Arbeiten ist hinsichtlich einer Gatekeeping-Theorie in diesem Band bereits thematisiert worden. Sie ist für verschiedene lateinamerikanische Autor/innen der Zeit anhand von Archivmaterialien belegt worden (vgl. Marling 2016; Einert 2018; Müller 2020). Auch auf Lispectors Rezeption im spanischsprachigen Lateinamerika scheint es zuzutreffen, dass viel davon abhing, ob bestimmte Schlüsselfiguren ihr Werk befürworteten und förderten. Monegal hatte dabei offenbar früh eine Strategie, die später in der gesamten englischsprachigen Welt und darüber hinaus sehr entscheidend wurde: „[...] recuperó su figura humana a través de encuentros y testimonios, bordeando la leyenda en la cual había comenzado a convertirse, comentando las relaciones entre esa leyenda y el contenido de sus textos" (Russotto 2013: 28). Ángel Rama hatte als weitere Schlüsselfigur, als akademischer Gatekeeper großen Einfluss auf die Verbreitung und Etablierung von Lispectors Werk im akademischen Kontext: „Rama, por su parte, difundió su obra en las aulas universitarias del continente, la latinoamericanizó, y la convirtió en referencia obligada en todos los congresos y encuentros internacionales dándole estatuto universal” (Russotto 2013: 28). Die 1970er Jahre markieren in Lateinamerika den Beginn einer breiteren internationalen Anerkennung des Werkes von Clarice Lispector, für die Frankreich in der darauffolgenden Phase eine Schlüsselrolle spielen sollte. 